\title{
Les lieux de dépôt des soutras bouddhiques en Chine : histoire et perspectives
}

\section{Jiang Haihong}

Traducteur : Coline Godard

\section{OpenEdition Journals}

Édition électronique

URL : http://journals.openedition.org/rbnu/934

DOI : $10.4000 /$ rbnu. 934

ISSN : 2679-6104

\section{Éditeur}

Bibliothèque nationale et universitaire de Strasbourg

\section{Édition imprimée}

Date de publication : 1 mai 2017

Pagination : 82-87

ISBN : 9782859230661

ISSN : 2109-2761

\section{Référence électronique}

Jiang Haihong, «Les lieux de dépôt des soutras bouddhiques en Chine : histoire et perspectives », La Revue de la BNU [En ligne], 15 | 2017, mis en ligne le 01 novembre 2019, consulté le 13 décembre 2020 URL : http://journals.openedition.org/rbnu/934; DOI : https://doi.org/10.4000/rbnu.934

\section{cc) (i) (2)}

La Revue de la BNU est mise à disposition selon les termes de la Licence Creative Commons Attribution - Pas d'Utilisation Commerciale - Partage dans les Mêmes Conditions 4.0 International. 


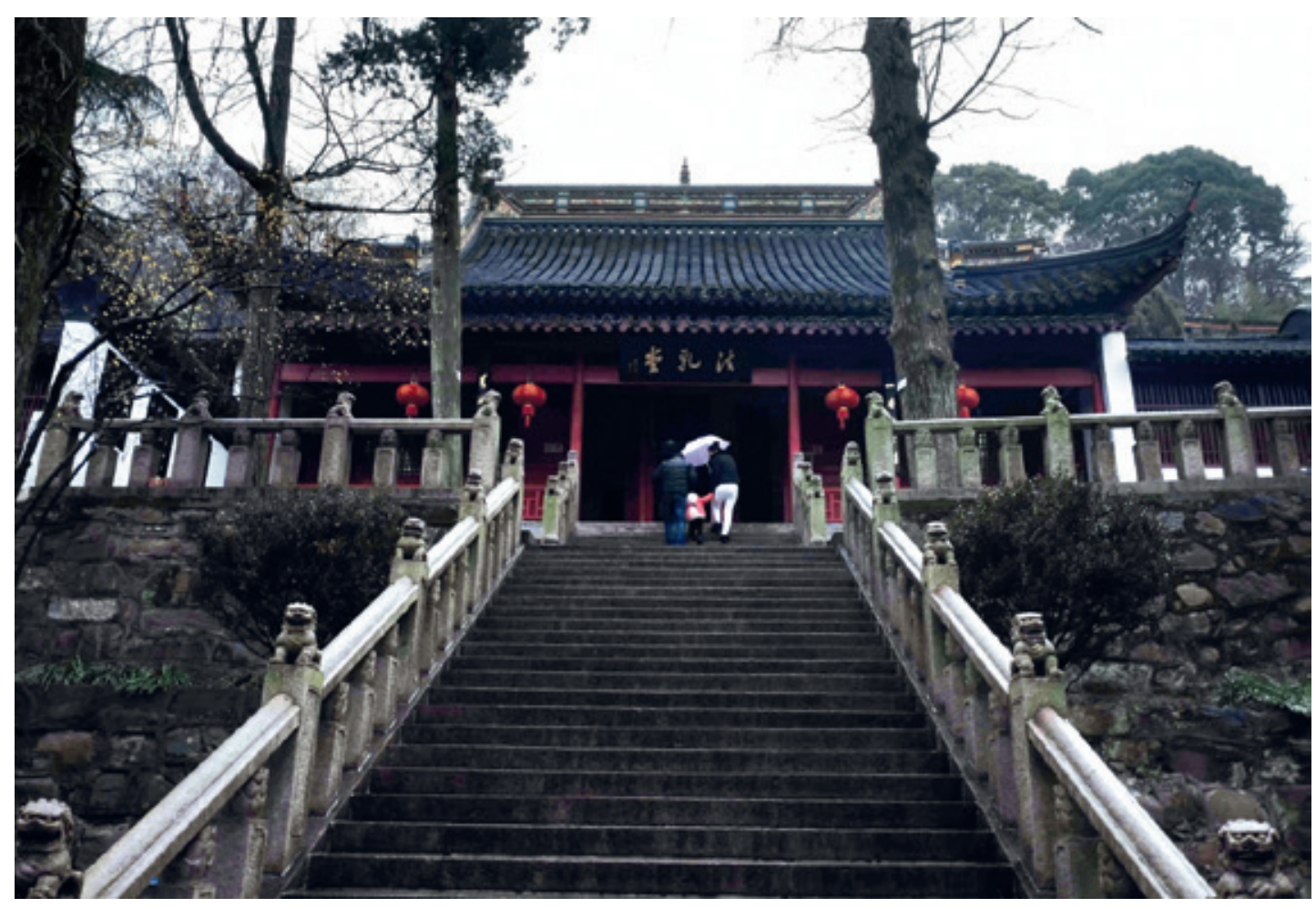

Une famille chinoise s'apprête à honorer le Bouddha au temple Guangiiao de Nantong, Jiangsu, Chine

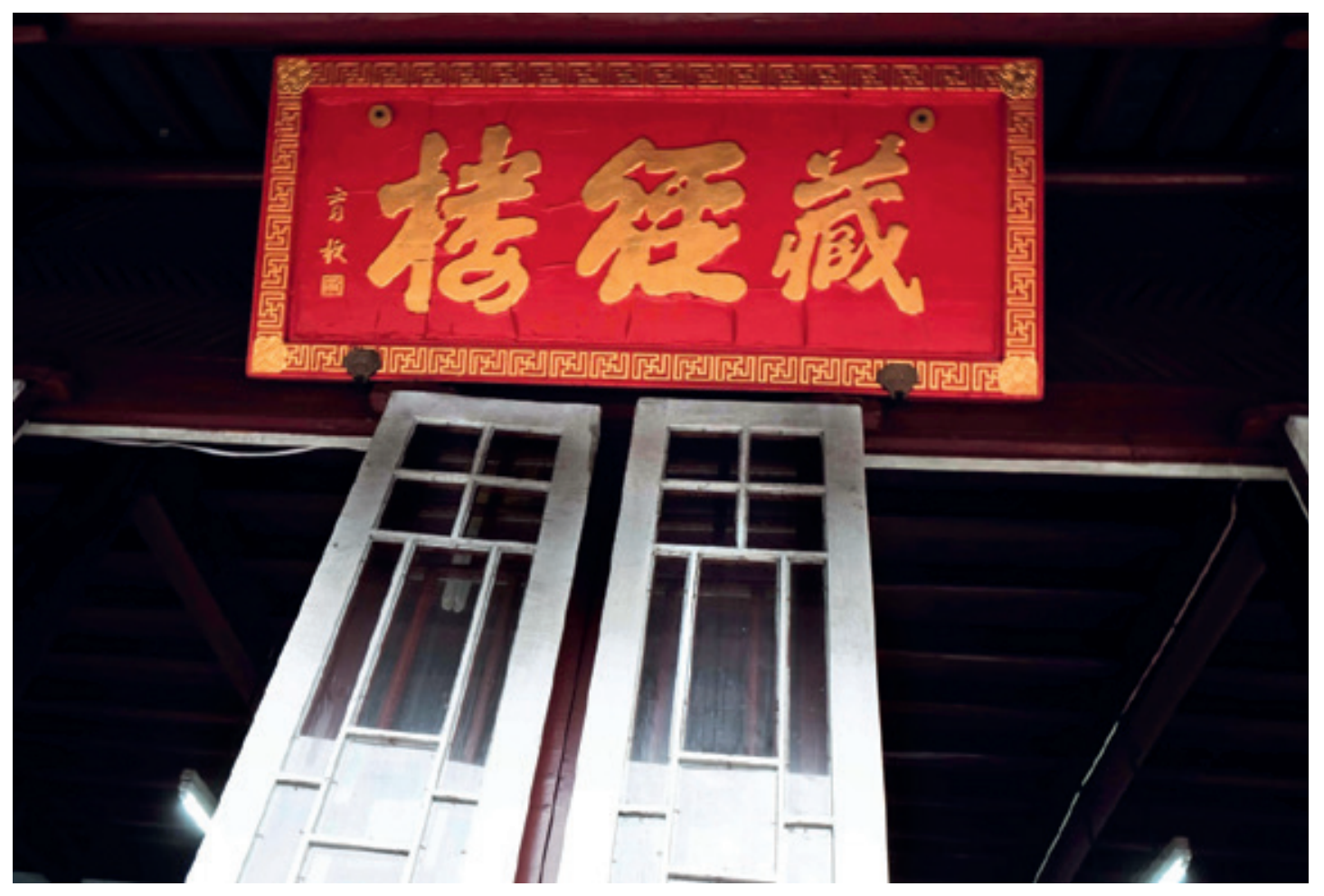

Lieu de dépôt de soutras bouddhiques du temple Guangjiao de Nantong, Jiangsu, Chine 


\section{LES LIEUX DE DÉPÔT DES SOUTRAS BOUDDHIQUES EN CHINE : histoire et perspectives

\author{
PAR JIANG HAIHONG
} \\ TRADUCTION FRANÇAISE COLINE GODARD}

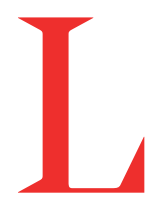

e bouddhisme est profondément ancré dans la culture chinoise. Vraisemblablement introduit en Chine sous la dynastie Han, aux premiers siècles de l'ère chrétienne, il s'est considérablement développé sous les dynasties du Nord et du Sud (420-589) pour atteindre son apogée avec la dynastie Tang (618-907). Cette expansion en Chine s'explique par le soutien affiché du gouvernement central, ou plus précisément des différents empereurs qui voyaient dans le bouddhisme un moyen efficace de forger les croyances du peuple et d'en réguler le comportement, en prônant l'accès à une vie meilleure dans l'au-delà grâce à une conduite exemplaire.

Le bouddhisme ainsi largement diffusé eut une incidence directe sur la construction de temples en Chine. Le premier monument érigé dans l'actuelle ville de Luoyang (province du Henan) marque le début d'une longue période, de 57 à 75, durant laquelle la majorité des édifices construits dans le pays étaient des temples bouddhistes. L'un des poèmes de $\mathrm{Du} \mathrm{Mu}$, de la dynastie Tang, propose une juste illustration de la prospérité du bouddhisme et de ses lieux de culte à cette période de l'Histoire : « Des quatre cent quatre-vingt temples des dynasties du Sud subsistent de nombreux pavillons surgissant de la brume ».

L'apparition des lieux de dépôt de soutras bouddhiques correspond à celle des temples, tout autant destinés à la collecte, la gestion et l'usage des Écritures bouddhiques qu'à la conservation et la vénération des trésors monastiques. Nos propos seront ici centrés sur la collecte et la diffusion des livres. Dès l'an 100, le temple était le lieu dédié à la traduction et à la lecture des Écritures bouddhiques. La construction de nouveaux temples et le développement des traductions et des publications de textes ont entraîné l'accroissement des bibliothèques monastiques et de leurs collections. Dès lors, ces bibliothèques ont mis en place une méthode encadrant les opérations de tri, de récolement, d'adaptation, d'acquisition et de catalogage. Les ouvrages collectés par les temples constituent aujourd'hui une large part du catalogue d'ouvrages anciens en Chine. À côté de cet objectif de collecte des textes canoniques, les bibliothèques monastiques de la Chine ancienne étaient entièrement ou partiellement ouvertes au public. Tout comme les lieux de dépôt de soutras bouddhiques, elles ont joué un rôle fondamental dans l'enseignement des moines et la diffusion de la doctrine bouddhiste parmi la population. Cet article proposera une brève introduction aux origines de la collecte d'ouvrages, puis examinera le lectorat et la condition actuelle des lieux de dépôt de soutras bouddhiques en s'appuyant sur les exemples des temples de Nantong (province du Jiangsu) qui, s'ils ne correspondent pas aux temples chinois typiques, illustrent toutefois convenablement l'état des temples monastiques sous l'aspect qui en est envisagé ici.

\section{Aux origines de la collecte d'ouvrages dans} les lieux de dépôt de soutras bouddhiques

Les ouvrages collectés par les temples sont généralement divisés en deux catégories : ceux traitant du bouddhisme et les autres ouvrages classiques. Selon Huang Jiannian ${ }^{1}$, les temples opèrent leur collecte principalement selon les critères suivants :

\section{Impressions officielles et publications à des fins} de collection :

De nombreuses et vastes collections d'Écritures bouddhiques ont été officiellement publiées et distribuées à différents temples, en particulier sous les dynasties de la Chine ancienne. 


\section{Transcriptions monastiques :}

Nombreux sont les moines qui copient les Écritures en gage de sincérité envers le Bouddha, un processus d'enrichissement personnel qui revêt pour eux une importance capitale. Il est courant de voir ces précieuses Écritures bouddhiques perdurer et se transmettre ainsi d'une génération à l'autre.

\section{Transcriptions de disciples et de fidèles :}

Au cours de la longue histoire de la Chine, de nombreux érudits et calligraphes de renom furent aussi des disciples du bouddhisme. À l'instar des moines, ils copièrent les Écriture pour illustrer leur foi et prouver leur respect envers le Bouddha. Ces ouvrages classiques constituaient ordinairement les collections précieuses des bibliothèques monastiques.

\section{Les ouvrages académiques individuels :}

Comme les ouvrages collectés par les temples sont généralement conservés dans de bonnes conditions et transmis de génération en génération, les bibliophiles traditionnels et les universitaires commencèrent à leur faire don de leurs propres ouvrages, contribuant ainsi à l'enrichissement des bibliothèques monastiques.

\section{Les dons issus de bibliothèques personnelles :}

Certaines bibliothèques monastiques ont pu enrichir leurs collections d'ouvrages précieux grâces aux dons issus de collections personnelles.

\section{Les ouvrages achetés :}

Les collections des bibliothèques monastiques ont également pu être enrichies grâce à l'acquisition de certains ouvrages indispensables.

\section{L'usage traditionnel des lieux de dépôt}

\section{de soutras bouddhiques}

En Chine ancienne, ceux-ci étaient accessibles aux moines comme aux citoyens ordinaires. Il est généralement admis que le lectorat des bibliothèques monastiques se divisait ainsi :

\section{Les moines :}

Pour les moines des temples, la fonction première de la bibliothèque monastique est de faciliter leurs études. La pratique de l'incantation fait partie de leur quotidien et est encouragée, de même que la lecture, à des fins d'enrichissement personnel.

\section{Les non-initiés :}

Les non-initiés sont ceux qui croient en Bouddha mais préfèrent s'instruire par eux-mêmes plutôt que de devenir moines ou nonnes. Ils ont cependant accès aux ouvrages des bibliothèques monastiques.

\section{Les bibliophiles et les érudits :}

Comme la doctrine bouddhiste préconise d'aider ceux qui en ressentent le besoin et entretient traditionnellement des liens avec les bibliophiles, la plupart des bibliothèques monastiques étaient ouvertes à ceux qui n'avaient pas les moyens d'acheter des livres. En cela, elles fonctionnaient sensiblement sur le même modèle que les bibliothèques publiques modernes.

\section{_L L'évolution des bibliothèques monastiques dans les temps modernes}

$\mathrm{Au}$ début des temps modernes, les bibliothèques monastiques oscillent entre développement et extinction, confrontées qu'elles sont à des difficultés liées à l'évolution des valeurs sociales et aux bouleversements techniques. Les lecteurs ont accès à de nouvelles sources d'information et se tournent de moins en moins vers les bibliothèques monastiques, dont le statut de bibliothèques publiques s'estompe peu à peu. Les moines eux-mêmes ont recours à d'autres méthodes pour cultiver leurs connaissances, fréquentant par exemple divers instituts monastiques, ou se tournant vers l'apprentissage et les discussions en ligne. Bon nombre de bibliothèques monastiques anciennes ne servent plus qu'à conserver Écritures et objets anciens, dans la limite d'espaces restreints et d'installations vétustes. Certains temples, peu nombreux, prennent cependant des mesures concrètes pour relever les défis de cette nouvelle ère. Ils agrandissent leurs bibliothèques et les modernisent en proposant air conditionné, ordinateurs connectés et vastes espaces de lecture. Des cours dominicaux et des projets d'enseignement autonome voient le jour, dans l'optique de rapprocher le peuple du bouddhisme ou au moins de l'intéresser aux temples et à la doctrine de cette religion. Mais pour satisfaire les non-initiés et ceux qui s'intéressent au bouddhisme en comptant sur un fonctionnement réactif, les bibliothèques monastiques ont encore beaucoup à faire. Plusieurs recherches académiques se sont aventurées sur ce terrain. Selon leurs auteurs, les points suivants doivent être pris en compte : 


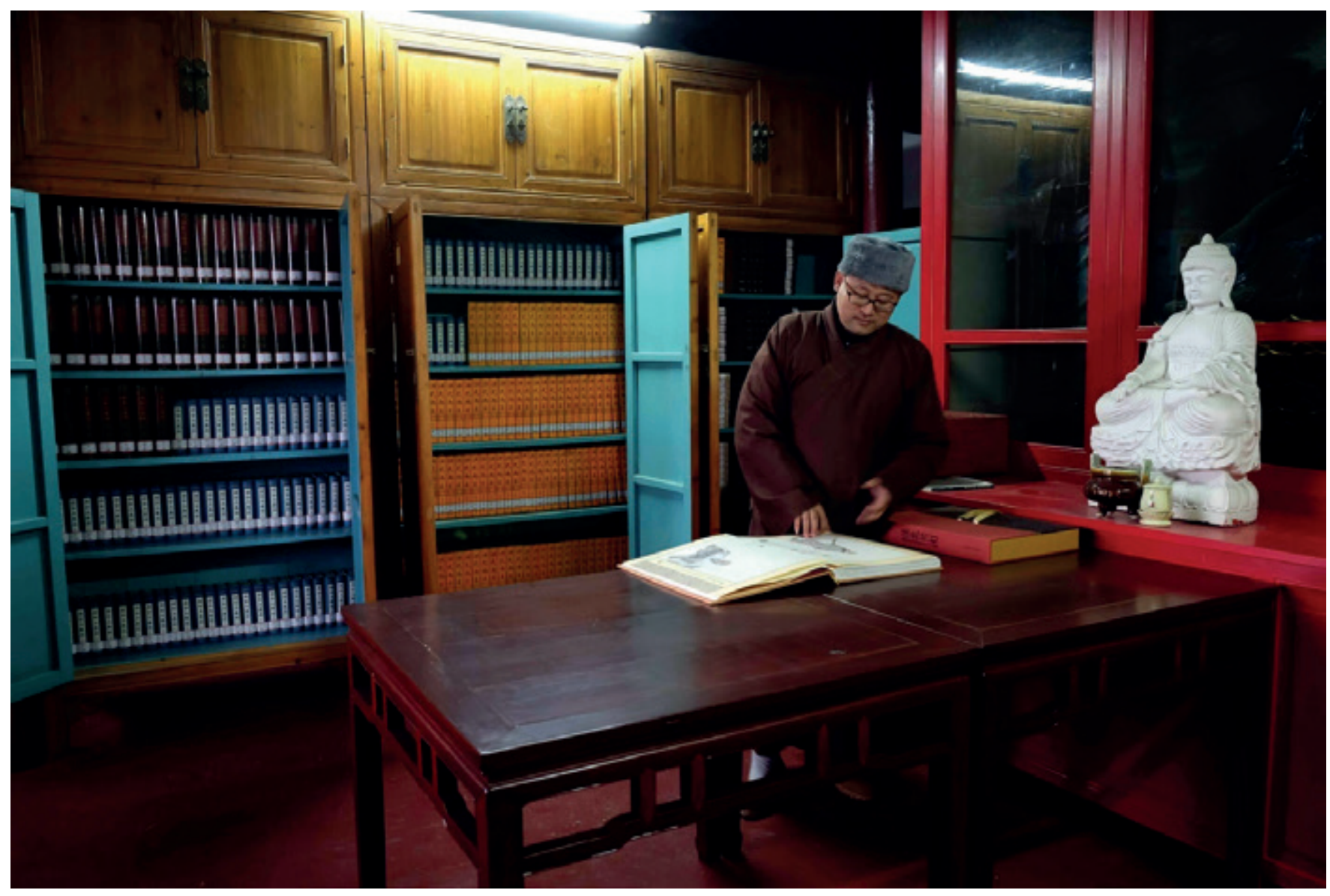

Un moine en pleine lecture dans l'ancien lieu de dépôt de soutras bouddhiques

Une équipe de direction aux domaines de compétences multiples :

Pour éviter de tomber en désuétude, les bibliothèques monastiques doivent de toute urgence se doter d'équipes de direction réellement efficaces, menées par des directeurs ayant une vision précise de l'évolution de ces lieux conformément à une feuille de route bien définie. Pour former ainsi une équipe performante, des compétences multiples sont nécessaires, aussi bien en termes de gestion des collections, de technologie de l'information et d'informatique, de communication ou de diffusion. Les membres de l'équipe responsables de chaque étape du travail garantissent la gestion efficace et productive de la bibliothèque. En cas de manque d'effectifs, les bibliothèques monastiques peuvent encourager le bénévolat, de la part des non-initiés et des fidèles. 



Les bibliothèques monastiques nouvellement équipées du temple Fahua, ville de Rugao, Jiangsu, Chine 


\section{Une gestion des collections optimale et régulièrement actualisée :}

À l'ère de l'échange et de la recherche d'informations en ligne pour les usagers, les bibliothèques monastiques doivent évoluer pour répondre à cette demande et mettre en œuvre leur connexion au réseau du service public. Des outils de recherche bibliographique informatisés, conformes aux normes nationales d'indexation et de catalogage, devraient dès lors faire leur apparition pour concrétiser le partage des ressources entre bibliothèques monastiques, mais aussi avec le réseau des bibliothèques publiques. Une maintenance quotidienne serait également nécessaire pour garder ces outils performants à jour. Il faudrait en outre engager une réflexion sur l'éventuelle mise en place de bibliothèques numériques.

\section{Des bibliothèques monastiques bien équipées et répon- dant à tous les besoins :}

Comme nous l'avons vu précédemment, les bibliothèques monastiques traditionnelles rassemblent des ouvrages chinois classiques ou centrés sur le bouddhisme, pour des usagers qui ont des profils et des objectifs variés. Modernisées, elles devraient par conséquent prendre cet aspect en compte en augmentant d'une part leurs collections de livres, en créant d'autre part des services spécifiques en fonction des groupes d'usagers. De la même manière que ce lectorat est divisé en catégories, les bibliothèques monastiques pourraient mettre en place différentes sections afin de répondre aux besoins de tous. La création de centres de recherche, de salles de lecture et d'amphithéâtres est à même de procurer un service de qualité. Les moines et chercheurs spécialistes du bouddhisme peuvent consulter des ouvrages classiques et précieux en salle de recherche, tandis que la salle de lecture est là pour les usagers manifestant un intérêt plus général pour le bouddhisme ou d'autres thèmes. Quant à l'amphithéâtre, il sert lors de lectures ou de séances de méditation, pour inviter l'abbé du temple ou d'autres moines éminents à présenter les textes bouddhiques. Les non-initiés et les fidèles peuvent être autorisés à participer à des séances de méditation dans l'amphithéâtre. Tous ces espaces doivent être équipés de l'air conditionné, d'accès en ligne et de machines de reproduction diverses (copieurs laser, ordinateurs, imprimantes et scanners, graveurs CD, appareils photo). Pour ces services, les usagers devraient naturellement s'acquitter des frais correspondants.

\section{Une communication entretenue avec, et soutenue par les collectivités locales :}

Les bibliothèques monastiques, qui s'affirment comme des lieux caritatifs, s'accordent pleinement avec les exigences des collectivités locales en termes de promotion culturelle. Elles doivent donc communiquer avec ces dernières et obtenir leur soutien pour que cette stratégie bénéficie au plus grand nombre. Les modes de coopération entre ces deux parties peuvent être variés, et comporter par exemple lectures et illustrations d'ouvrages classiques chinois à l'attention du grand public. Cette coopération est mutuelle et bénéfique. Les bibliothèques pourront alors mieux s'intégrer dans la société, survivre et se développer dans l'ère moderne. En contrepartie, les collectivités locales peuvent jouer un rôle moteur pour l'intégration des ressources de ces bibliothèques dans le réseau public, les aidant ainsi à mieux fonctionner.

Avec une histoire de plus de 2000 ans, le bouddhisme représente en Chine un patrimoine culturel considérable et revêt une importance capitale dans l'évolution de l'humanité. De plus amples recherches s'imposent sur le développement $\mathrm{du}$ bouddhisme, recherches dont l'une des pierres angulaires est la sauvegarde et l'avenir des bibliothèques monastiques.

\section{ORIENTATIONS BIBLIOGRAPHIQUES ${ }^{2}$}

- Huang, Jiannian, « Research on Depositories of Buddhist Sutras of Chinese Ancient Temples ", in Library and Information, $\mathrm{n}^{\circ}$ 3, 2011

- Huang, Jiannian, «An Open Study on Depositories of Buddhist Sutras of Chinese Ancient Temples ", in Library and Information Service, $n^{\circ} 9,2011$

- Jiang, Weiqiao, History of Buddhism in China, Zhonghua Book Co, 2015

- Shen, Chunqiao, «A Tentative Study on the Development and Utilization of Depositories of Buddhist Sutras », in Zhejiang Archives, $\mathrm{n}^{\circ} 12,2003$

- Zhang, Zhongmin, « On the Past and Current Situations of Collected Books in Chinese Ancient Buddhist Temples ", in Library Theory and Practice, $\mathrm{n}^{\circ} 4,2010$

\section{NOTES}

1- Voir les références en bibliographie.

2- La majorité des articles référencés sont écrits en chinois sans versions anglaises. Les titres traduits peuvent présenter de légères différences par rapport aux titres originaux. 